\title{
Dynamic Scenarios Transformation in Software System Design in Software Architectural Pattern through MVC
}

\author{
Dr. G Manjula, Harshitha G S and Dr. G Mahadevan
}

\begin{abstract}
In the real word many software performance prediction methodology are available such that the design decisions are clearly understandable by the stakeholders. To improve the existing software performance Palladio component model methodology is used by little improving algorithm of the problem context which in turn reflected in the system design. The software architectural patterns are the best tool in measuring the quality. There are different types of architectural patterns like distributed systems, interactive systems etc. There are two types of communicatingstructuresknown as Model View Controller (MVC) then Presentation Abstraction Controller (PAC).
\end{abstract}

Keywords - Software System Design, MVC.

\section{INTRODUCTION}

In the real word many software performance prediction methodology are available such that the design decisions are clearly understandable by the stakeholders. To improve the existing software performance Palladio component model methodology is used by little improving algorithm of the problem context which in turn reflected in the system design. The software architectural patterns are the best tool in measuring the quality. There are different types of architectural patterns like distributed systems, interactive systems etc. There are two types of communicatingstructuresknown as Model View Controller (MVC) then Presentation Abstraction Controller (PAC). Model View controller is more efficient tool and incorporated with the programming languages like UML, Eclipse, Object oriented patterns languages, java, etc.

\section{A. SCOPE OF THIS WORK}

The interactive architectural pattern is definitely having further clarifications in the quality assurance and the performance. Dynamic Scenario transformation is one of the challenging force and the measuring design by using performance prediction tool called PCM in turn the dynamic scenarios of the architectural pattern varies a lot. This method is recommendable such that easily tracing the solution, limited constrains, and scenarios, simulations and mathematical

Dr. G Manjula, Professor\& HOD, Department of Computer Science \& Engineering Sri Sairam College of Engineering, Anekal, Bengaluru, Karnataka, India.

Harshitha G S, Department of Computer Science \& Engineering, BMSIT\& M, Avalahalli, Doddaballapura Main Road, Yelahanka, Bengaluru, Karnataka, India.

Dr. G Mahadevan, Principal, Annai College of Engineering, Kovilacheri, Thanjavur, Tamil Nadu, India. transformation can be easily verified. By just modifying the Design decisions like Class, diagram. Sequence diagram, CRC card, set of constraints etc. in the software architectural pattern (specified), the interrelated module and the set of entity can be further improved and the quality of the design system performance, fault tolerance, efficiency and the cost (pertain to the requirement specification) can be enhanced compared to the existing systems.

The present development is bounded with a set of constrains. The proposed methodology is implemented in the two standard data sets. The comparative result recommends the performance of the system surly improved and still more practical ground level exercise have been done. The agile process in the software engineering motivated the change in the existing system. Some of the self-questions on the correlated modules are tested and verified with different $b$ services.

The architecture language (ADL) is the main challenging task to analyze the architecture synthesis and maintenance of the architecture

Palladio component model is a hierarchical model, which inherits the sub model called Meta model. The Meta model is used in measuring the quality of the execution, which in turn reflects on the cost,and the quality of the framework.

Palladio component model is a normal state configuration structure where programming procedure will interact effectively with the quality related issues in the existing system design. The product procedure aimed at calculating quality in the execution, unwavering quality then estimation charge will be utilized in product by using different design programming language like simulation. The simulation is also one of the apparatuses where the system designs execute third party or the outermost layer in the stakeholders then in turn calculating measurements. PCM is the process for evaluating the performance prediction by using the architectural patterns and utilized in numerous approaches. Forecast techniques designed for execution then unwavering quality based programming frameworks stand discreet constrained further once now a while utilized as a part of industry Component engineers who deliver segments that are amassed by programming designers and conveyed by framework allocators. The differing data required for the forecast of additional useful properties is in this way spread among these designer parts. PCM can likewise be utilized in light of the distinctive information set, where the behavioral aptitudes of information are information uprightness. The every conduct of information can be put into arrangement 
outline and follow out. The general procedure of design the MVC for performance is shown in the figure1.There are basically three units interrelated with each other like design inputs, design activities and design outputs. The area of the system is architectural design, which in turn related with the interface design and database design. By modifying the architectural design using MVC, the efficiency of the system can be improved. The architecture will be same, when most of the systems domain same. The technology behind the domain may also be same. To fulfill the customer s requirements the application product will in turn bounded by core architecture of structure. The structural design of any classification stays designed by various architectural styles. The architectural patterns are means of reusing the object oriented design methods. This method relates towards programming designs demonstrated through the Palladio Component Model. It underpins quantifiable execution, dependability, besides charge forecast then correlated with each other, reached out towards extra reckonable excellence standards of programming designs. Through including, another segment display in the middle of the every framework remains other compelling in computing also effortlessly reasonable in several professional solicitations.

\section{REVIEW OF LITERATURE}

Approach depends on programming execution forecast [34, 1]; design based programming unwavering quality examination [17], and seeks based programming building [22]. Firmly related methodologies are

1. Imperativecentred methodologies 2. Met heuristiccentered methodologies.

Imperative based Methodologies: Xu et al. [number sequence] show partial-robotized way of dealing with and configuration and plan change scheduled in prototype typical mode. In view of LQN show, execution issues (e.g., restricted access, extended behaviors) standby recognized an initial step. At that point, rules containing execution learning are connected to the identified issues. The approach cannot distinguish change denied, and a portion upgrades usage of segments, managing discovery parts. Proposes will not achievable to outline arrangements. Instance, may be difficult to accelerate specific segment usage to achieve specific administration time in view of intrinsic many-sided quality.

McGregor et al. [28] built up the ArchE outline work. ArchE helps the product engineer amid plan to make models prerequisites. It makes structural models, gathers necessities (in type of situations), gathers the data expected to break down the quality criteria for the prerequisites, gives the assessment apparatuses to modifiability or execution investigation, and proposes enhancements. Contrasted with seek entire outline progresses step-wise in view of standards. It does not bolster unwavering quality and just components a straightforward execution display. The engineering part therefore, flexibility introduced cannot promptly recognize.

Cortellessa et al. [11] input era for programming execution investigation, which goes for efficiently assessing execution forecast, comes about utilizing step-wise fortification. Depending, discovery normal execution issue designs (execution against examples) in the execution show. There is no support to naturally fathom a recognized hostile to design, and proposal engineering applicants.

Bondarev et al. [5] present Deep Compass outline work for configuration space investigation of implanted frameworks. The system depends on the ROBOCOP part show. It utilizes a Pareto investigation to determine the sentencing for ideal execution and low expenses for various design hopefuls. Along these lines, execution measurements every design hopeful expenses of every competitor. Determination design competitors and gives to proposing new hopefuls.

\section{EXISTING SYSTEM VARIATIONS}

In Adaptive Programming Model-View-Controller (MVC) depicts two varieties of MVC: an aloof model and a dynamic model. The inactive model is utilized when one controller controls the model solely. The controller adjusts the model and afterward educates the view that the model has changed and ought to be invigorated the model in this situation is very autonomous of the view and the controller, which implies that there are no methods for the model to report changes in its state. The HTTP convention is a case of this. There is no straightforward route in the program to get no concurrent upgrades from the server.

It shows the view and reacts to client enter, yet it does not identify changes in the information on the framework. Just when the client unequivocally asks for the example then framework cross-examined for changes.

Passive prototypical model: During this, model whatever the changes or moderations done by the view it will not be updated in the model. The model will always store the data that is in the repository for further processing. Due to this deviation in the existing system the model will not been updated. This scenario of operation in the transformation of the CBSE is called passive model.

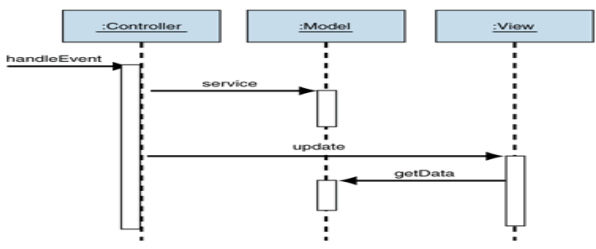

Fig. 3.1 Dynamic scenarios variation in the Software architecture

Active model: During the active model state, the view model will perform the task sharpen by the controller as a controlling the system. The model will stores the current data (while processed in the view model and in turn send new updated moderations in the Repository) once the process is done by the view.

\section{CASE STUdy 1}

\section{A. ZEND FRAME WORK IN MVC PROGRAMMING DESIGN}

The methodology applied in two study cases with certain constraints. Investigation of subspace mixture model and various distance measure techniques in class diagram.

The UML-MVC Logical Model 
The coherent model explained comprises of stereotyped standard UML charts and is proposed to show the product segments to be created for each of the three layers of the MVC design [8] and additionally the connections among. In particular, the model is organized into the accompanying UML graphs:

Model Class Diagram (MCD): an UML class graph demonstrating the classes that take an interest in the Model layer and execute the application business rationale and information persistency.

View Class Diagram (VCD): an UML class graph speaking to the customer and server pages in the View layer. These pages have the obligation of displaying information and substance to the client and empowering client connection with the framework. This chart likewise models:

Classes making part of the Controller layer;

Associations between client collaborations (e.g., the "Submit" of a type of a View page) and techniques for classes of the Model that are in control to serve;

Associations between characteristics of classes of the View (which relate to information gave/asked for by the application) to techniques for classes of the Model that oversee.

Navigation connects between pages of the View.

3. One UML Sequence Diagram depicting the associations between the different segments of the framework and their state moves amid the execution of complex client exercises and $b$ exchanges. What's more, this graph is utilized to depict the route ventures between pages of the View that are connected with the diverse return estimations of the execution of the strategies for Model classes.

The subsequent general model is autonomous of the particular innovations decided for the usage of the application, consequently it is a PIM in the MDA design, yet it is as of now adequately definite to control the application's improvement group. The model can be utilized to make the application with any execution innovation in view of the MVC design. On a trial premise, the source code is utilized the Java Server Faces framework [15].

The major technical challenging issues, which are largely unresolved in the dynamic scenario, are

1. Intra-subject variability.

2. Small sample size problems

3. Single sample per person for training

4. Unconstrained conditions

5. Subject's non cooperation

Pattern recognition problems can be solved essentially by solving the distance or similarity measures technique to handle the problem. Automatic recognition is the increasing attention in identifying the face recognition process.

Proposed Algorithm for various distance measure techniques is:

Step 1: Defining the architecture with state diagram.

Step 2: Identifying basic styles in heterogeneous architecture based on system design features.

Step 3: Mapping the state diagram to Markov model.
Step 4: Integrating Markov models to create an overall Markov model.

Step 5: Creating the separate sets for each style and enforcing limitations.

Step 6: Creating the transition probability matrix.

Step 7: Calculating the visit number of each state in Markov model.

Step 8: Evaluating the efficiency of the model.

Software architecture pattern is the ever growing research area, the slight modification if adopted in the design part of the pattern, the drastic changes can be evolved which in turn reflects on the performance of the system. First, a uniform algebraic approach is in cultivated to improve on previous formal work. The characteristics of the component are modified by writing extraordinary close program strategy linguistic with normal notation. Another, guaranteeing the component life span by replacing the existing component, which in turn results in the result of the architecture. Third, reconfigurations and working out are obviously associated by observance sequence. This is for the reason that the method delivers semantics to a specified architectural complete the algebraic structure of a different platform.

Thus, scenario era is much similar to programming testing: you cannot demonstrate that you have an adequate number of experiments, hover you can decide a time when the expansion of new experiments is giving unimportant change to the product as shown in the Figure 4.4

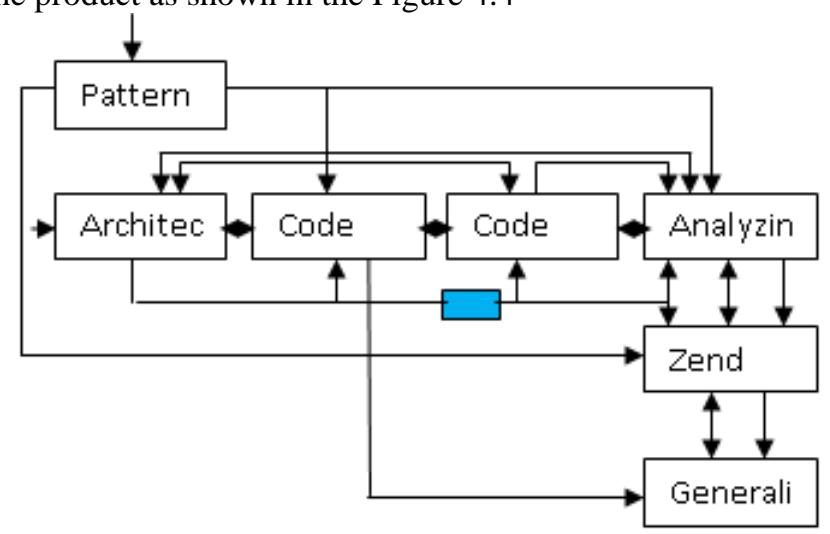

Figure 4.1 M-Accurate withZend framework in MVC

\section{B. Deciding the correct arrangement of components in the transformation}

In immense accentuation that MDD puts the different components, a fascinating inquiry are "while takes components produced an adequate amount of consequences to satisfactorily validate evaluation. On the other hand, put another way: when would it be a good idea for one to quit creating different circumstances. Here conceivable responses have basicsolutionis: "the point at which you come up short on assets". The more mind boggling, and more important answer includes thinking about back the investigation procedure. One can quit producing transformation are expansion of another set-up complicates outline.

One method for minimizing the quantity of scenarios required (once more, on similarity validation), software architecture gathering developments of proportionality Data 
Types, talked about in previous chapter 2.3. In any case, simply produces another complication of the given case study. Accentuation on modules of states decide how to design union, might figure out if transformation suitably gathered keen onmodules. On the off chance that both the transformations in the above cases be $\mathrm{P}$ and $\mathrm{Q}$, grouped a design, rather ought to similar state, before they should apportioned no less than two unmistakable auxiliary parts. On the off chance that these parts contain usefulness, which is insignificant as per the general inclination of scenarios aPas well as Q simply develop extra state, bring about cooperation segments are considered aQis distributed. During the off chance that, then again, the parts contain just the usefulness apropos as per the general inclination of scenarios an $\mathrm{B}$, then ought to see the accompanying example in examination: a similar arrangement of basic segments influenced by togetherstatesand Q, different segments stand influenced states are not considered.

Additional state of situation about the issue of consequence modules is as shown in the Figure 4.5 space specialists ought to group scenarios a similar way. On the off chance that they do not, they have extra, certain scenarios as a main priority, and these must be evoked.

A strategy space for handler interface structural design. The design space architecture associated with set of instructions, which will styles the architectural substitutions of the software. The system majorly focuses on the interactive user interface for some functions. The functional dimensions of the design space architecture which reflect on the scenario is external event handling, user customizability, user interface adaptability across the devices, computer system organization, basic interface class and application portability across user interface styles.

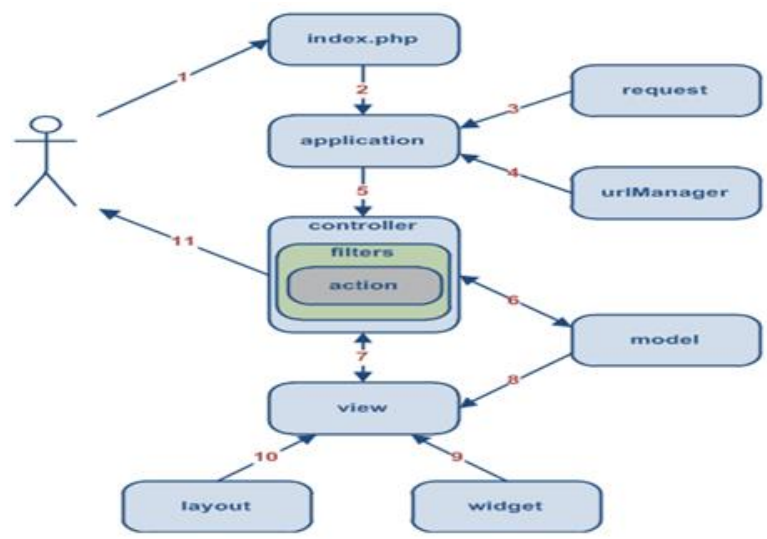

Fig. 4.2 M-Accurate implementation model

Zend framework in MVC programming design

1. A client makes a demand with the framework and the $b$ server handles the demand by executing the bootstrap script index.php

2. The bootstrap scripts make an application case and runs it.

3. The application acquires gritty client ask for data from an application segment named ask.

4. The Application decides the asked for controller and activity with the assistance of an application segment named
urlManager.

5. The Application makes an occurrence of the asked for controller to further handle the client asks. The controller class. It then makes and executes channels, connected with this activity. The activity is executed on the off chance that it is permitted by the channel.

6. The activity peruses a Post model who's ID is one from the database.

7. The activity renders a view named shoe with the Post show.

8. The view peruses and shows the properties of the Post display.

9. The view executes a few gadgets.

10. The view rendering result is installed in a design.

11. The activity finishes the view rendering and shows the outcome to the client.

\section{This approach includes:}

Assessing the diverse in Software Engineering Paradigms as for their fittingness to incorporate growing new procedures as shown in the Figure 4.6, techniques, forms that consider security as a component of the product improvement life cycle; Tool Support/characterize a Suitable Example; Transfer of security learning/travel look into results to standard framework advancement.

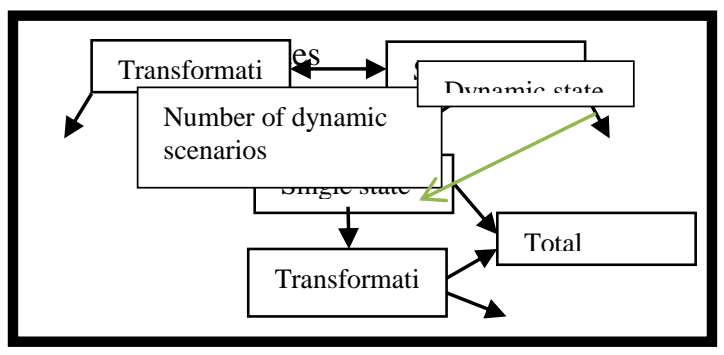

Fig. 4.3 M-Accurate Evaluation model

Zen framework improves the performance, as MVC pattern design in corporate

Zend Framework 2 utilizes a module framework to sort out fundamental application-particular code inside every module. The Application module gave by the skeleton is utilized to give bootstrapping, blunder and directing design to the entire application. It is normally used to give application level controllers to, say, the landing page of an application, hover are not going to utilize the default one gave in this instructional exercise as need collection rundown to be the landing page, which will live in own particular component.

\section{PERFORMANCE PREDICTION AFTER INSERTING NUMBER OF MODULES}

There are different types of performance prediction after inserting the modules in the software architectural design issues such that some of the major networking is

1. Queuing network: The performance of any system with reference to the architectural pattern, by imposing to the architectural style, imposing to the architectural patterns and related to the patterns is more precisely explained by Denning \& Buzman, 1978; Jain 1991; Kleinrocks 1975; lazowska et al. 
1984; Menasce et al. 2004.

2. Markov chains: By understanding the rules of Markov chain, the performance of the system can also be easily traced out. The complete history related to the Markov chain is described by Trivedi 1982 .

3. Layered Queuing network:Theadvance tool set used in the queuing network, which is in turn related to the architectural pattern like, layered. The layered architectural pattern will improve the flexibility of the system but decreases the performance by Rolia \& Sevcik 1995; Woodside et al. 1995.

4. Stochastic Petric nets: There are different methodologies are bounded to predict the performance of a system. Here majorly focused on the networks and their efficiency prediction in the system by Bause \& Kritzinger 1996.

5. Stochastic process algebras: The performance prediction using model driven development can also be evaluated by algebraic process, graphical transformation, win and loss strategies also been used by Hermanns et al 2002.

6. Software with their Software Performance Engineering (SPE): The software performance can be evaluated by using different software tool set like model transformation, component interface, Scenario based, evaluation based, ATL etc.by Smith 1990 and 2002.

7. Software Performances Engineering (SPE): The software performance engineering community will yield to performance modeling notations which in turn yield to transformation Analytical models briefly explained by Bassamo et al 2004 .

8. Performance related extension to UML: The approach considered in this are defacto standard Modeling languages (UML SPT profile) which briefly analyzed by OM 2005.

9. UML marte profile: The different tool set are been used to calculate the enactment of a system. The scenario based, Component Based Software Engineering (CBSE) and many more tools are been described by OMG 2006.

10. Meta model of the performance domain: The Meta model transformation also been used in the performance prediction. The majorly many literature survey have been evaluating the performance of the system efficiently with limited constrains given by Cortellessa 2005. Which is in turn established the SPE-MM.

The some of the strategy prediction when the M-Accurate approach is applied to the case study Zend framework are

In system architecture, identified the passive mode and active mode of MVC strictly based on the ADL. The CBSE uses a different model named CONCRETE prototypical between the model and view. By means of CONCRETE component is interpreted between two component models then the proposed system behavioral structure varies as shown in the figure 3.1. The general principles of the architectural design as discussed in turn need to modify, during the proposed system many set of problem raised as discussed earlier.

\section{FUTURE SCOPE AND ENHANCEMENT}

In late time, a few new strategies have been produced at a fast pace. A portion of the headways in persistent years, new techniques have been produced at a quick pace. A portion of the progressions in persistent improvement strategies have been centered on correlation and differentiating nature of Evolutionary Algorithms and Gradient based techniques. Truly, an Evolutionary calculation is one of the best techniques accessible for subordinate - free enhancement on higher dimensional issues. This approach will most likely make contrast in the current framework, while the measuring measurements programming stage changes in every application. Approach applies to programming designs demonstrated with the Palladio Component Model. It bolsters quantitative execution, dependability, and cost forecast and can be stretched out to other quantitative quality criteria of programming models. By including another segment demonstrate in the middle of the every framework is more viable in measuring and effectively appropriate in any business application. In Software life cycle, the two key exercises included are Requirements Engineering and programming architecting specialists are emphasing on mapping and change of necessities to programming design, hover the absence of powerful arrangement is yet common.

In this area, introduce the M-ACCURATE approach. The name M-ACCURATE originates from an acronym for' Model a Configurable Code generator Unified with Requirements Analysis Techniques'. As it suggests, necessities assume an essential part in both PIM displaying and stage choice. The key thought is to catch practical and non-useful necessities into isolated relics, a PIM and a stage design separately, and go along with at the downstream of the advancement

\section{REFERENCES}

[1] Karl J. Lieberherr, College of Computer Science, North-eastern University Cullinane Hall, Boston, MA 02115 "Metrics of Graph Abstraction for Component-Based Software Architecture" Los Angeles, California USA March 31-April 02 ISBN: 978-0-7695-3507-4

[2] Science of Computer Programming - Special issue on "Applications of graph transformations (GRATRA 2000) " Volume 44 Issue 2, August 2002 Publisher: Elsevier North-Holland, Inc. Amsterdam, The Netherlands, The Netherlands ISSN: 0167-6423 doi:10.1016/S0167-6423(02)00036-9

[3] Guo Wei, Xiong Zhong-Wei, Xu Ren-Zuo, "Metrics of Graph Abstraction for Component-Based Software Architecture," csie, vol. 7, pp.518-522, 2009 WRI World Congress on Computer Science and Information Engineering, 2009.Articles:Visual Design of Software Architecture and Evolution based on Graph Transformation https://doi.org/10.1109/CSIE.2009.697

[4] Eclipse.org. ATLAS Transformation Language (ATL). http://www.eclipse.org $/ \mathrm{m} 2 \mathrm{~m} / \mathrm{at} 1 /$.

[5] A. Billig, S. Busse, A. Leicher, and J. G. Süss. "Platform Independent Model Transformation Based on TRIPLE". In Middleware'04: Proceedings of the 5th ACM/IFIP/USENIX International Conference on Middleware, pages 493-511, 2004.

https://doi.org/10.1007/978-3-540-30229-2_26

[6] D. Ayed and Y. Berbers. "UML Profile for the Design of Platform-Independent Context-Aware Applications. In MODDM" 06: Proceedings of the 1st Workshop on Model Driven Development for Middleware, pages 1-5, 2006. https://doi.org/10.1145/1169086.1169090

[7] OMG. Meta Object Facility (MOF) 2.0 Query/View/Transformation Specification Version 1.0. ttp://www.omg.org/docs/formal/08-04-03.pdf, 2008.

[8] S. Link, T. Schuster, P. Hoyer, and S. Abeck. "Focusing Graphical User Interfaces in Model-Driven Software Development". In ACHI'08: Proceedings of the 1st International Conference on Advances in Computer-Human Interaction, pages 3-8, 2008 https://doi.org/10.1109/ACHI.2008.16 